\title{
Interactive comment on "Constructing a complete landslide inventory dataset for the 2018 Monsoon disaster in Kerala, India, for land use change analysis" by Lina Hao et al.
}

Lina Hao et al.

hao_In@qq.com

Received and published: 21 September 2020

Thanks for all the constructive comments and suggestions. We have revised the manuscript and responded to all the comments. Details of the responses were in the supplement document.

Comment 1: "The introduction explores comprehensively the available literature although the objectives in mind are not very clear."

Response: Thanks for your constructive comments. Our main objective as stated in the last sentences of the introduction: "The main objective of the study is to develop

Printer-friendly version

Discussion paper 
a comprehensive event-based landslide inventory database for the 2018 Monsoon in Kerala, that can be used to analyze to what extent these landslides were affected by land use changes." In order to make the introduction section more clearly, some redundant description were deleted, while others related to our methodology description were added. Details of the revisions are as following:

1) The original Line 55 to Line 59 of "such as supervised classification (Lacroix et al., 2013), Object Based Image Analysis (OBIA)(Behling et al., 2014;Casagli et al., 2016; Keyport et al., 2018; Lahousse et al., 2011; Mohan Vamsee et al., 2018), Markov random fields (Lu et al., 2019; Qin et al., 2018), random forests (Stumpf and Kerle, 2011), support vector and other machine learning methods (Lei et al., 2019) or a combination of various algorithms (Aksoy and Ercanoglu, 2012; Li et al., 2016; Lu et al., 2011; Stumpf and Kerle, 2011)." was deleted. The related references were also deleted.

2) Other irrelevant contents such as "UAV, SAR, InSAR" in the original line 59 and 60 were also deleted.

3) "The availability of multi-sourced and multi-temporal high resolution satellite images (HRSI) on the Google Earth platform with 3D viewing capabilities (Crosby, 2012; Fisher et al., 2012) offered major advantages for landslide inventory mapping (Mohammadi et al., 2018). Many authors have generated landslide inventories using the Google Earth platform (Rabby and Li, 2019; Sato and Harp, 2009; Fiorucci et al., 2011; Borrelli et al., 2015). It has also proven to be possible to map event-based landslides by comparing images before and after the event using Google Earth history Viewer (Xu et al., 2014a, 2014b). However, recognizing and mapping specific types of landslides such as rainfall triggered shallow landslides over large areas can be still challenging when using automated techniques. Field verification is only feasible for a limited number of landslides, as it is time and labor intensive, and many landslides may be difficult to access. Therefore, visual image interpretation using HRSI from different time periods may be the best solution. Landslide mapping and classification requires mapping experience and the availability of high resolution images in three-dimension views, either

Interactive

comment
Printer-friendly version

Discussion paper 
using stereo images, or oblique views such as in Google Earth, allowing to recognize the specific diagnostic features (Soeters and van Westen, 1996; Zieher et al., 2016)." was added in the introduction section. Also the related references were added in the reference list.

4) The order of some sentences was also modified in paragraphs in the introduction section.

Interactive

comment

5) The related references was added in the bibliographic list.

comment 2: "In the section 3.1 Landslide mapping, the authors should consider to clarify the following points: Please, indicate what LULC means (land use land cover?)."

Response: LULC means Land Use and Land Cover in section 3.1 Landslide mapping. The detail of LULC was supplemented in the revisions in the last paragraph of the introduction section as follows: "This manuscript focuses on the generation of the dataset consisting of a detailed landslide inventory with land use/land cover (LULC) information for two periods: shortly before the event, and almost a decade older."

comment 3: "Despite the difficulty in identifying landslide from photos and sometimes establish elementary diagnostic features, it will be very helpful if the authors explain how superficial slide (SS) can be distinguished from debris flow (DF); and the latter from rock fall (RF), considering the different sources at stake."

Response: Thank you for this comment. We have added the following sentences to description how to discrimination of SS, DF and RF. Also the related reference for the discrimination was added in the reference list. "Based on the diagnostic features described in Soeters and van Westen (1996), debris flow (DF) features were differentiated from shallow landslides (SS) by the presence of a runout zone, often reaching to the nearest stream, which is not the case for SS. Rock fall features (RF) can be differentiated from the other two processes as they occur on very steep and bare rocky slopes"

comment 4: "Bearing in mind that the images have too coarse resolution for a proper 
Response: Landslides were mapped from multi-temporal sub-meter high-resolution images in Google Earth's history viewer, which allowed us to differentiate the three types of landslides according to their discernible image features. Details of discrimination of the three types of landslides were mentioned in the response to comment 3.

comment 5: "Could the authors give comprehensive explanations why the majority of the landslides, in the 2018 monsoon event, occurred mainly within forest areas? This behavior is mentioned several times, included in the 6 Discussion and conclusions Taking this aspect into account, the authors might include previously, in the section 2.1 Study area, a description regarding the topographic and orographic characteristics of the entire zone. As you know, the slope is a critical issue for this type of movements in addition to a major triggering event. At a same time, those areas are not attractive for human activities."

Response: This paper is mainly focusing on the generation of the comprehensive landslide inventory after the 2018 monsoon event. We are writing a follow-up paper in which we analyzed the land use / land cover changes and evaluate these changes. As this paper is focused on the data collection part, we do not give such a comprehensive explanation in this manuscript. The topographic and orographic characteristics of the study were given in the "2.1 Study area" section. However, we would like to answer to this comment briefly here. There are two reasons that the majority of the landslides occurred mainly within forest areas in the 2018 monsoon event.

1) The land use map of 2010 from KSDMA (Kerala State Disaster Management Agency) used in this research as a reference to distinguish natural forest from forest plantation, indicates that the forest coverage rate (natural forest and forest plantation) is $76.51 \%$ in this area. The forest coverage in steep areas is even higher, which are more susceptible to landslides. Also, a large part of Kerala is covered by forest (natural forest or forest plantation) confirmed by literatures (Kumar 2005; Roy et al. 2015; Kale

Interactive comment
Printer-friendly version

Discussion paper 
et al. 2016; Sudhakar Reddy et al. 2016; Fox et al. 2017; Ramachandra and Bharath 2019).

ESSDD

2) Physiographically, the west of Kerala consists of coastal plains covered mainly with built up areas and agriculture such as paddies. The eastern part of Kerala is within the Western Ghats with rugged mountains and deep valleys covered with forest and forest plantation. Most of the landslides triggered in the 2018 monsoon event thus were in the eastern mountainous parts of Kerala.

comment 6: "Please, check bibliographic list. Lines 314 and 316: the references Sahana (2019); Ramachandra and Bharat (2019) are not listed."

Response: Thank you for pointing this out. We checked all the references and added the missing references. The references of Sahana (2019), Ramachandra and Bharat (2019) were supplemented in the bibliographic list.

Please also note the supplement to this comment: https://essd.copernicus.org/preprints/essd-2020-83/essd-2020-83-AC1supplement.pdf

Interactive comment on Earth Syst. Sci. Data Discuss., https://doi.org/10.5194/essd-2020-83, 2020. 